\title{
Downregulated expression of PHLDA1 protein is associated with a malignant phenotype of cholangiocarcinoma
}

\author{
PO ZHAO, XIAOYING LI, YALI LU and LIN LIU \\ Department of Pathology, Chinese People's Liberation Army General Hospital, Beijing 100853, P.R. China
}

Received July 4, 2014; Accepted April 24, 2015

DOI: $10.3892 / 01.2015 .3316$

\begin{abstract}
Cholangiocarcinoma is one of the most aggressive types of malignancy, and is associated with poor patient prognosis. Recent findings suggest that a decrease in pleckstrin homology-like domain family A, member 1 (PHLDA1) expression is significant in the induction of cell migration and tumor invasion. The clinicopathological significance of the expression of PHLDA1, and its potential correlation with the expression of CD133 in cholangiocarcinoma have remained to be elucidated. In the present study, PHLDA1 protein expression was investigated by immunohistochemical analysis of 218 cholangiocarcinoma tissue samples, as well as 30 para-neoplastic and 20 normal bile ducts. The expression status of PHLDA1 and CD133 was determined, and these results were analyzed against the age, gender, tumor location and size, histological grade, clinical stage and overall mean survival time of the patients. The expression of PHLDA1 protein was markedly decreased in $35.3 \%$ of cholangiocarcinomas, compared with that of the para-neoplastic and normal cholangiocytes. Carcinomas with loss of expression of PHLDA1 were significantly correlated with the tumor site $(\mathrm{P}=0.001)$, histological grade $(\mathrm{P}=0.020)$ and clinical stage $(\mathrm{P}=0.0001)$, but not with age $(\mathrm{P}=0.085)$, gender $(\mathrm{P}=0.456)$ or size $(\mathrm{P}=0.413)$, respectively. Kaplan-Meier survival analysis indicated that the loss of expression of PHLDA1 was significantly correlated with the overall survival time ( $\log$ rank=193.861; $\mathrm{P}=0.0001)$. Furthermore, the expression of PHLDA1 was found to be inversely correlated with the expression of CD133 $(\gamma=-0.142$; $\mathrm{P}=0.036)$. These findings suggested that the decreased expression of PHLDA1 may be significant in the carcinogenesis and progression of cholangiocarcinoma, and may represent a novel adjunct marker of disease prognosis.
\end{abstract}

Correspondence to: Professor Po Zhao, Department of Pathology, Chinese People's Liberation Army General Hospital, 28 Fuxing Road, Beijing 100853, P.R. China

E-mail: zhaopo301@163.com

Key words: cholangiocarcinoma, pleckstrin homology-like domain family A, member 1, immunohistochemistry, prognosis, pathology

\section{Introduction}

Cholangiocarcinoma is one of the most aggressive types of malignancy, and is associated with poor patient prognosis $(1,2)$. This is due to the fact that at the time of diagnosis, when the disease becomes clinically evident, the majority of cancers of the biliary tract will have outgrown the limits of curative resection. Complete resection represents the only potential curative therapy for all types of biliary tract neoplasm (3). The morbidity of bile duct cancer varies according to country, area and ethnicity (4). In China, the survival rate of this kind of rare carcinoma is decreased compared with in Western countries, with a tumor-free 5-year survival rate of $\sim 13 \%(3,5)$. There are various underlying causes of cholangiocarcinoma, including hepatitis virus infection, particularly hepatitis $\mathrm{C}$ virus, chronic biliary infections of intestinal origin, primary desmoplastic cholangitis, bile duct lithiasis, infection by Clonorchis sinensis and environmental carcinogens, which represent potential pathogenesis factors for Chinese patients (3). Advances in biological research, particularly those regarding mutation-independent activation of the Hedgehog pathway (6), have aided understanding of the carcinogenesis and progression underlying this rare tumor. Furthermore, these results provide an opportunity for the development of targeted therapies for the treatment of biliary tract cancer. However, early pathological diagnosis remains difficult in such highly desmoplastic, submucosal, infiltrating types of cancer. Currently, sensitivity for the diagnosis of cholangiocarcinoma is only $\sim 30 \%$ for cytology, and $40-70 \%$ for combined brush cytology and biopsy, rendering negative results virtually useless $(2,3)$. Therefore, novel early diagnostic tools and therapeutic techniques for this disease are urgently required.

Pleckstrin homology-like domain family A, member 1 (PHLDA1) encodes a 401-amino acid protein, which comprises a central pleckstrin homology domain common to proteins involved in intracellular signaling or as constituents of the cytoskeleton (7-9), a central polyglutamine tract and 2C-terminal regions rich in proline-glutamine and proline-histidine repeats. The PHILDA1 gene is expressed in a wide range of normal and cancer tissues $(10,11)$. PHLDA1 function varies with cell type and context, with several studies reporting proapoptotic (11-14) or antiproliferative roles (15). PHLDA1 expression is induced by external stresses, for example heat shock $(13,14)$, and may be modulated by the insulin-like growth factor I (16) and extracellular-regulated 
kinase pathways (16). The expression of PHLDA1 protein was previously characterized and compared with that of proposed markers of intestinal stem cells in the human small and large intestine (17). Sakthianandeswaren et al (17) found that PHLDA1 was coexpressed with leucine-rich repeats containing G-protein coupled receptor 5 ( $\operatorname{Lgr} 5)$ in the previously reported intestinal epithelial stem cells in murine crypt base cells, and further determined that PHLDA1 expression was a marker of putative epithelial stem cells and contributed to intestinal tumorigenesis. PHLDA1 is overexpressed in human intestinal tumors of all stages, and may be involved in cell migration, as suggested by the increased staining and nuclear relocalization of the protein at the invasive front of intestinal carcinomas. Accordingly, colon cancer cells demonstrate significantly reduced migratory behavior in response to PHLDA1 suppression (17), and in skin tumors, PHLDA1 is recognized as a follicular stem cell marker for differentiation between basal cell carcinomas and trichoblastomas (18). PHLDA1 protein is constitutively expressed by nevi in vivo (11), which raises the possibility that PHLDA1 expression may contribute to the benign nature of these tumors, maintaining the regulation of growth and apoptotic sensitivity to the loss of survival signals provided by adjacent keratinocytes. Therefore, the progressive downregulation of PHLDA1 expression associated with malignant transformation may contribute to the loss of these characteristics in melanoma. Downregulation of PHLDA1 protein has also been reported to be a significant predictor of poor prognosis for breast cancer patients (10). PHLDA1 is a substrate of Aurora A (19), which directly phosphorylates PHLDA1, resulting in its degradation. PHLDA1 also negatively regulates Aurora A, via the promotion of Aurora A degradation, thereby forming a feedback loop. PHLDA1 upregulation therefore antagonizes Aurora A-mediated oncogenic pathways, thereby revealing PHLDA1 degradation as a mechanism by which Aurora A promotes biliary tract malignancy (20). Therefore, although the mechanisms underlying the downregulation of PHLDA1 expression remain to be elucidated, the loss of PHLDA1 expression may contribute to the development of apoptosis resistance in cholangiocarcinomas. The progressive loss of PHLDA1 expression in cholangiocarinomas may induce dysregulated cell growth and apoptosis resistance in these tumors. PHLDA1 is a putative tumor suppressor in cholangiocarcinoma and therefore the significance of the loss of expression of PHLDA1 in cholangiocarcinoma requires further investigation.

The present study aimed to identify the possible role of PHLDA1 protein in the progression of human cholangiocarcinoma by investigating its expression in 218 samples of cholangiocarcinoma.

\section{Materials and methods}

Patients and specimens. Two-hundred and eighteen cholangiocarcinoma tissue samples from a cohort of patients who had undergone surgery for cholangiocarcinoma, 30 samples of the corresponding para-neoplastic bile duct tissue and 20 samples of normal bile ducts with inflammation were retrieved from the archives of the Department of Pathology, Chinese People's Liberation Army General Hospital (Beijing, China). All specimens were from patients who had undergone surgery between July 1998 and December 2006. The specimens were $10 \%$ neutral formalin-fixed, paraffin-embedded and stored in the archives. Each tissue specimen was histologically evaluated by at least two experienced pathologists. The carcinoma patients included 130 males and 88 females aged 17-73 years old (mean age, 53.6 years; median age, 56.0 years). The tumor locations were as follows: 53 intrahepatic, 103 perihilar and 62 distal cholangiocarcinomas. Tumor grading and staging was performed by applying World Health Organization (2000) and Union for International Cancer Control (1997) criteria (1). The tumor grading was as follows: 69 cases at grade 1, 91 cases at grade 2 and 58 cases at grade 3; and staging; 7 cases at stage I, 95 cases at stage II, 88 cases at stage III and 28 cases at stage IV. Ethical approval for this study was not required by The Committee of Medical Ethics of Chinese People's Liberation Army Hospital as the experiments conducted were not associated with the privacy, impairment or treatment of the patients.

Immunohistochemical analysis. All reagents were purchased from Zhongshan Golden Bridge Biotechnology Co., Ltd (Beijing, China) unless otherwise stated. Paraffin-embedded tissue sections (4- $\mu$ m thick) were cut, dewaxed in xylene and rehydrated in a graded ethanol series. Subsequently, endogenous peroxidase activity was blocked by immersing the sections in $3 \%$ hydrogen peroxide in methanol for $10 \mathrm{~min}$ prior to rinsing in running water. Sections were then immersed in boiling $0.01 \mathrm{M}$ EDTA buffer ( $\mathrm{pH}$ 8.0) in a pressure cooker, which was sealed and brought to full pressure for $2 \mathrm{~min}$. The pressure cooker was then depressurized and cooled under running water, prior to removal of the lid and flushing out of the hot buffer with cold water from a running tap. The cooled sections were washed twice in phosphate-buffered saline (PBS) prior to immunohistochemical analysis. The sections were incubated at $4^{\circ} \mathrm{C}$ overnight in a humidified chamber with monoclonal mouse antibody against human PHLDA1 (Santa Cruz Biotechnology, Inc., Dallas, TX, USA) at 1:50 dilution and polyclonal rabbit antibody against human CD133 (Abcam Inc., Cambridge, MA, USA) at 1:100 dilution in blocking solution. Following exposure to the primary antibodies, sections were incubated with the polyperoxidase-anti-mouse/rabbit immunoglobulin $\mathrm{G}$ for $20 \mathrm{~min}$ using the standard non-biotin PV-6000 Polymer Detection System (Zymed Laboratories Inc., San Francisco, CA, USA). The sections were then washed in water, counter-stained with Mayer's hematoxylin for $1 \mathrm{~min}$ at room temperature, dehydrated, cleaned and mounted. Paraffin blocks of human breast ductal carcinoma tissues were used as positive controls. Negative controls were sections treated analogously, but $0.01 \mathrm{M}$ PBS was substituted for the primary antibodies. For immunohistochemical evaluation of PHLDA1 and CD133 expression, cytoplasmic and nucleic labeling of tumor cells was classified as positive. In scoring expression of PHLDA1 and CD133 protein, both the extent and intensity of staining were considered, in accordance with a study by Hao et al (21). The intensity of immunopositivity was scored as follows: Negative, 0; weak, 1; moderate, 2; strong, 3. While the extent of positivity was scored as follows: $<5 \%, 0 ;>5-25 \%$, $1 ;>25-50 \%, 2 ;>50-75 \%, 3 ;>75 \%$, 4 , of cells in the respective lesions. The final score was obtained by multiplying the intensity and the extent of positivity scores, yielding a potential 
Table I. Association between expression of PHLDA1 or CD133 and clinicopathological features.

\begin{tabular}{|c|c|c|c|c|c|c|}
\hline \multirow{2}{*}{$\begin{array}{l}\text { Clinicopathological } \\
\text { feature }\end{array}$} & \multicolumn{2}{|c|}{ PHLDA1 } & \multirow[b]{2}{*}{ P-value } & \multicolumn{2}{|c|}{ CD133 } & \multirow[b]{2}{*}{ P-value } \\
\hline & - & + & & - & + & \\
\hline Age, years & & & 0.085 & & & 0.395 \\
\hline$\geq 60$ & 33 & 44 & & 33 & 44 & \\
\hline$<60$ & 44 & 97 & & 70 & 71 & \\
\hline Gender & & & 0.373 & & & 0.407 \\
\hline Male & 49 & 81 & & 58 & 72 & \\
\hline Female & 28 & 60 & & 45 & 43 & \\
\hline Tumor site & & & 0.001 & & & 0.068 \\
\hline Intrahepatic & 29 & 24 & & 22 & 31 & \\
\hline Perihilar & 35 & 68 & & 44 & 59 & \\
\hline Distal & 13 & 49 & & 37 & 25 & \\
\hline Tumor size, $\mathrm{cm}$ & & & 0.413 & & & 0.003 \\
\hline$\leq 2$ & 33 & 70 & & 60 & 43 & \\
\hline$>2 \leq 5$ & 35 & 56 & & 37 & 54 & \\
\hline$>2$ & 9 & 15 & & 6 & 18 & \\
\hline Grade & & & 0.020 & & & $<0.001$ \\
\hline 1 & 16 & 53 & & 45 & 24 & \\
\hline 2 & 34 & 57 & & 42 & 49 & \\
\hline 3 & 27 & 31 & & 16 & 42 & \\
\hline TNM & & & $<0.001^{\mathrm{a}}$ & & & $<0.001^{\mathrm{a}}$ \\
\hline I & 2 & 5 & & 5 & 2 & \\
\hline II & 22 & 73 & & 57 & 38 & \\
\hline III & 33 & 55 & & 35 & 53 & \\
\hline IV & 20 & 8 & & 6 & 22 & \\
\hline Mean survival, months & 11.6 & 25.4 & $<0.001$ & 31.2 & 16.9 & $<0.001$ \\
\hline
\end{tabular}

${ }^{a}$ TNM I-II vs. TNM III-IV. PHLDA1, pleckstrin homology-like domain family A, member 1.

range of $0-12$. Scores of $\geq 4$ were defined as a positive expression pattern, while scores of $<4$ were recorded as negative.

Statistical analysis. Fisher's exact test, Pearson's $\chi^{2}$ test, Spearman's correlation coefficient test for trends in proportions and the Kaplan-Meier method with the Log rank test or Cox regression method for univariate or multivariate overall survival analysis were used to assess the associations between PHLDA1 or CD133 expression and pathological indices. $\mathrm{P}<0.05$ was considered to indicate a statistically significant difference.

\section{Results}

PHLDA1 expression is downregulated in cholangiocarcinoma tissues. PHLDA1 protein was expressed diffusely in the cytoplasm and nuclei of discrete cholangiocytes in all 30 para-neoplastic and 20 normal bile ducts (Fig. 1). In carcinoma, PHLDA1 was expressed diffusely in the cytoplasm and nuclei of cancer cells in 141 out of 218 cases $(64.7 \%$; Fig. 2). The expression of PHLDA1 was low or negative in the remaining $77(35.3 \%)$ cases of carcinoma. The majority of poorly-differentiated cancer cells were negative for PHLDA1 protein expression (Fig. 3). There was a significant difference in PHLDA1 immunopositivity between cholangiocarcinomas and para-neoplastic or normal bile ducts $(\mathrm{P}<0.0001)$. In addition, strong PHLDA1 nuclear staining was observed at the invasive margin (Fig. 4).

PHLDA1 expression is negatively correlated with, tumor site and histological grade. In the present group of 218 cholangiocarcinoma samples, PHLDA1 expression was negatively correlated with tumor site $(\mathrm{P}=0.001)$, grade $(\mathrm{P}=0.020)$ and stage $(\mathrm{P}=0.0001)$, but not with age $(\mathrm{P}=0.085)$, gender $(\mathrm{P}=0.373)$ or tumor size $(\mathrm{P}=0.413)$ (Table I). Follow-up data revealed that there was a significant difference in overall mean survival time between patients with PHLDA1-negative carcinomas (11.6 months) and those with PHLDA1-positive carcinomas (25.4 months; $\log$ rank=193.861; $\mathrm{P}=0.0001 ;$ Fig. 5). The result of multivariate analysis by Cox regression indicated that PHLDA1 expression was an independent prognostic factor $(\mathrm{P}=0.009)$.

Association between PHLDA1 and CD133 expression. In the present group of 218 cholangiocarcinoma samples, $52.3 \%$ of cases were CD133 positive. CD133 expression was correlated 


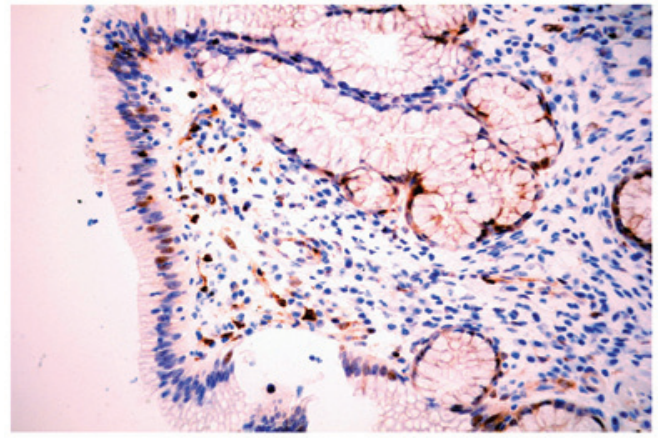

Figure 1. Expression of PHLDA1 in normal bile ducts. PHLDA1 was positively expressed in the cytoplasm and nucleus of indiscrete cholangiocytes. (magnification, x400). PHLDA1, pleckstrin homology-like domain family A, member 1.

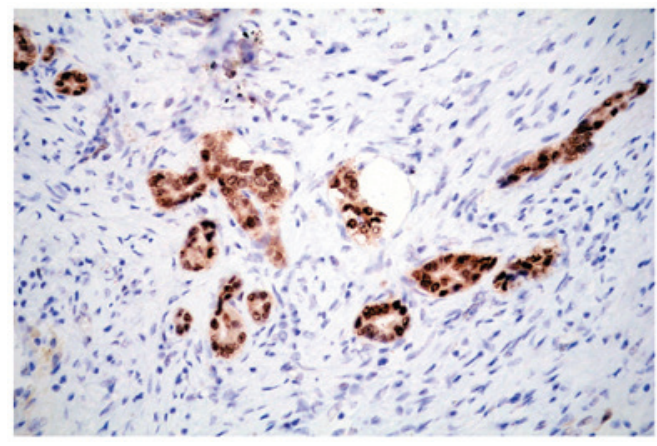

Figure 4. Expression of PHLDA1 in cholangiocarcinoma. Strong PHLDA1 nuclear staining was observed at the invasive front (magnification, $\mathrm{x} 400$ ). PHLDA1, pleckstrin homology-like domain family A, member 1.

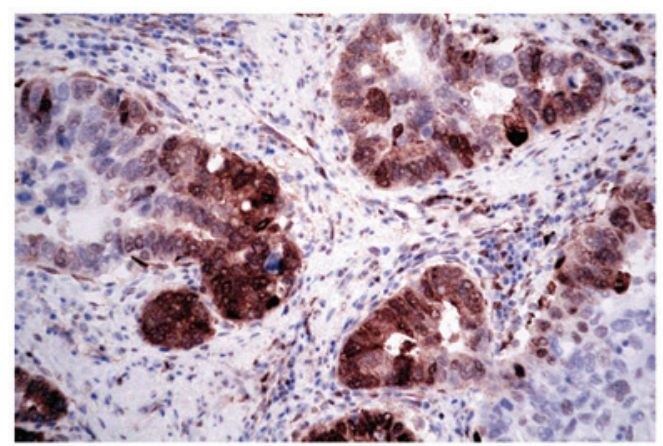

Figure 2. Expression of PHLDA1 in cholangiocarcinoma. PHLDA1 was positively expressed in the nucleus and cytoplasm of cancer cells in moderately to well-differentiated cholangiocarcinoma. (magnification, x400). PHLDA1, pleckstrin homology-like domain family A, member 1 .

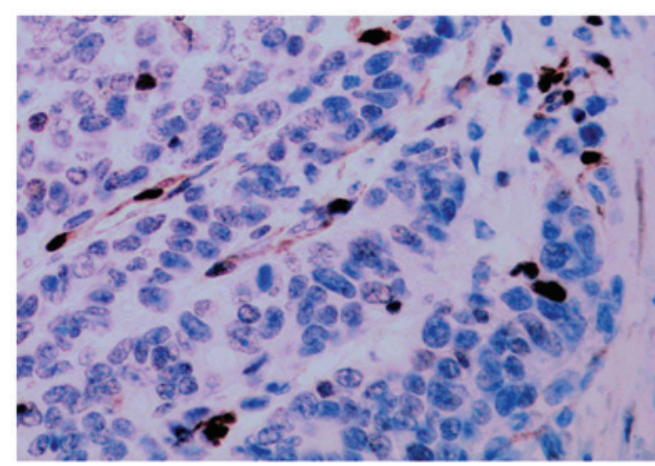

Figure 3. Expression of PHLDA1 in cholangiocarcinoma. PHLDA1 expression was negative in poorly differentiated cholangiocarcinoma. (magnification, x400). PHLDA1, pleckstrin homology-like domain family A, member 1 .

with tumor size $(\mathrm{P}=0.003)$, grade $(\mathrm{P}=0.0001)$, stage $(\mathrm{P}=0.0001)$ and overall mean survival time $(\mathrm{P}=0.0001)$, but not with tumor site $(\mathrm{P}=0.068)$, age $(\mathrm{P}=0.395)$ or gender $(\mathrm{P}=0.407$; Table I $)$. Frequently, when PHLDA1 expression was negative, CD133 was found to be positive in the cancerous glands of cholangiocarcinoma. Of the cholangiocarcinomas with high expression of CD133, PHLDA1 expression was only detected in $58.2 \%$ of cases (67/115), whereas of the carcinomas with low expression of CD133, PHLDA1 was expressed in $71.8 \%$ of cases $(74 / 103)$. A significant inverse association was detected between the expression of PHDA1 and CD133 ( $\gamma=-0.142 ; \mathrm{P}=0.036)$.

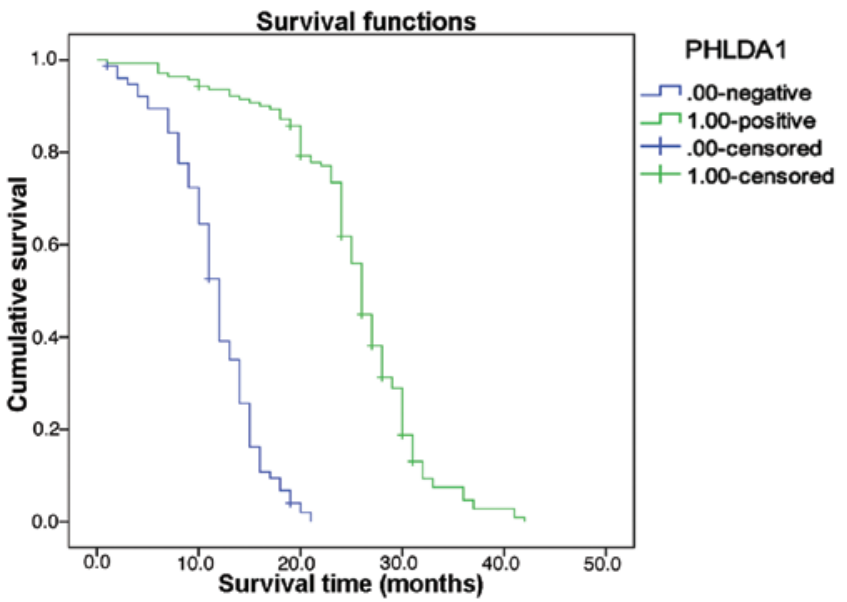

Figure 5. Kaplan-Meier survival analysis by PHLDA1 status $(n=218)$. The $\mathrm{y}$-axis represents the percentage of patients; the $\mathrm{x}$-axis, their survival in months. The green line represents PHLDA1-positive patients, showing a trend of better survival than the blue line, representing PHLDA1- negative patients. Mean overall survival time was 25.4 months for the PHLDA1- positive group and 11.6 months for the PHLDA1- negative group (Log rank=193.861; $\mathrm{P}=0.0001$ ).

\section{Discussion}

Sakthianandeswaren et al (17) identified PHLDA1 as a putative marker of epithelial stem cells in the human adult small and large intestine. In the colon and rectum, PHLDA1 protein was expressed in undifferentiated columnar cells limited to the crypt base. This distribution of PHLDA1-expressing cells in the human intestine closely resembles that of Lgr5 in the mouse intestine (17). PHLDA1 was constitutively expressed in human intestinal adenomas and the majority of carcinomas. A total of 218 cases of cholangiocarcinoma with follow-up data were analyzed in the present study and the results indicated that PHLDA1 was positively expressed in $64.7 \%$, and negatively expressed in $35.3 \%$ of carcinomas. Furthermore, the loss of PHLDA1 expression was correlated with the histological degree $(\mathrm{P}=0.0001)$ and clinical stage $(\mathrm{P}=0.0001)$. This was in contrast to the previous results in colorectal cancer by Sakthianandeswaren et al (17), in which no correlation between PHLDA1 staining and adenocarcinoma grade or clinical stage was observed, suggesting that PHLDA1 may have a significant role in the evolution and development of cholangiocarcinoma. Enhanced staining and nuclear relocalization 
of the PHLDA1 protein was also observed at the invasive front of the cholangiocarcinomas, similarly to the results in colorectal cancer reported by Sakthianandeswaren et al (17), which also suggested a novel role for PHLDA1 in cell migration. However, the mechanisms underlying the differential functions of nuclear and cytoplasmic expression of PHLDA1 remain to be elucidated. Notably, the polyglutamine tract in PHLDA1 is a feature common to several transcription factors, suggesting a potential role as a transcription factor or coactivator (22). Follow-up data revealed a significant difference in overall mean survival time between the PHLDA1-negative (11.6 months) and PHLDA1-positive cholangiocarcinomas (25.4 months) (Log rank=193.861; $\mathrm{P}=0.0001)$. The result of multivariate analysis by Cox regression indicated that PHLDA1 expression was an independent prognostic factor $(\mathrm{P}=0.009)$. The results of the present study also correspond with those of previously published results in breast cancer (10), melanoma (11) and oral squamous cell carcinomas (23). It has been suggested that PHLDA1 expression may be a potential prognostic factor in cholangiocarcinoma.

CD133 is an important marker in a variety of tumor stem cells (24-26). In the present study, CD133 expression was positive in $52.3 \%$ of cholangiocarcinoma cases. Investigation of the expression of CD133 protein in the 218 cholangiocarcinoma with follow-up data, indicated that CD133 expression level was positively correlated with histological degree $(\mathrm{P}=0.0001)$ and clinical stage $(\mathrm{P}=0.0001)$. Furthermore, expression of the CD133 protein was significantly associated with overall survival $(\mathrm{P}=0.0001)$, suggesting that CD133 expression may be a potential prognostic factor for poor prognosis in cholangiocarcinoma. The results above correspond with those of previous reports regarding cholangiocarcinoma from Japan (27), Thailand (28) and a research group from Xian, China (29), in which the positive rates of CD133 in cholangiocarcinoma were 48.3, 67.6 and $74.0 \%$, respectively, and CD133 expression was considered to be a potential prognostic indicator. Subsequently, the potential association between PHLDA1 and CD133 was investigated. In the present study, PHLDA1 expression was demonstrated to be inversely associated with clinicopathological features, including clinical stage, histological grade and poor prognosis in cholangiocarcinoma. These results also indicated that PHLDA1 may be inversely correlated with CD133 $(\mathrm{P}=0.0001)$, implying loss of PHLDA1 function may occur in high CD133-expressing cancer cells. However, the association between these two genes identified is descriptive rather than causative, and determination of a clear functional association required further investigation.

In conclusion, PHLDA1 expression may be downregulated in malignant phenotypes of cholangiocarcinoma. The detection of PHLDA1 and CD133 expression may, to some extent, reflect the biological behavior of cholangiocarcinoma cells, aiding the selection of appropriate chemotherapy and molecular targeting therapies.

\section{Acknowledgements}

The authors would like to thank their colleagues in the Department of Pathology, Chinese People's Liberation Army General Hospital (Beijing, China) for their technical assistance.

\section{References}

1. Khan SA, Davidson BR, Goldin R, et al: Consensus document. Gut 51 (Suppl 6): VI1-VI9, 2002.

2. Gores GJ: A spotlight on cholangiocarcinoma. Gastroenterology 125: 1536-1538, 2003.

3. Zhao P, Lu Y, Zhong M, Liu L and Li B: Inverse correlation of aberrant expression of fragile histidine triad (FHIT) protein with cyclin D1 protein and prognosis in Chinese patients with cholangiocarcinoma. Acta Oncol 47: 1557-1563, 2008.

4. Heron DE, Stein DE, Eschelman DJ, et al: Cholangiocarcinoma: The impact of tumor location and treatment strategy on outcome. Am J Clin Oncol 26: 422-428 2003.

5. Wu ZF, Wu XY, Zhu N, et al: Prognosis after resection for hepatitis B virus-associated intrahepatic cholangiocarcinoma. World J Gastroenterol 21: 935-943, 2015.

6. Berman DM, Karhadkar SS, Maitra A, et al: Widespread requirement for Hedgehog ligand stimulation in growth of digestive tract tumors. Nature 425: 846-851, 2003.

7. Haslam RJ, Koide HB and Hemmings BA: Pleckstrin domain homology. Nature 363: 309-310, 1993.

8. Ingley E and Hemmings BA: Pleckstrin homology ( $\mathrm{PH})$ domains in signal transduction. J Cell Biochem 56: 436-443, 1994.

9. Saraste M and Hyvonen M: Pleckstrin homology domains: A fact file. Curr Opin Struct Biol 5: 403-408, 1995.

10. Nagai MA, Fregnani JH, Netto MM, et al: Down-regulation of PHLDA1 gene expression is associated with breast cancer progression. Breast Cancer Res Treat 106: 49-56, 2007.

11. Neef R, Kuske MA, Pröls E and Johnson JP: Identification of the human PHLDA1/TDAG51 gene: Down-regulation in metastatic melanoma contributes to apoptosis resistance and growth deregulation. Cancer Res 62: 5920-5929, 2002.

12. Park CG, Lee SY, Kandala G, Lee SY and Choi Y: A novel gene product that couples TCR signaling to Fas (CD95) expression in activation-induced cell death. Immunity 4: 583-591, 1996.

13. Hossain GS, van Thienen JV, Werstuck GH, et al: TDAG51 is induced by homocysteine, promotes detachment-mediated programmed cell death and contributes to the development of atherosclerosis in hyperhomocysteinemia. J Biol Chem 278: 30317-30327, 2003.

14. Hayashida N, Inouye S, Fujimoto M, et al: A novel HSF1-mediated death pathway that is suppressed by heat shock proteins. EMBO J 25: 4773-4783, 2006.

15. Oberst MD, Beberman SJ, Zhao L, et al: TDAG51 is an ERK signaling target that opposes ERK-mediated HME16C mammary epithelial cell transformation. BMC Cancer 8: 189, 2008.

16. Toyoshima Y, Karas M, Yakar S, Dupont J, et al: TDAG51 mediates the effects of insulin-like growth factor I (IGF-I) on cell survival. J Biol Chem 279: 25898-25904, 2004.

17. Sakthianandeswaren A, Christie M, D'Andreti C, et al: PHLDA1 expression marks the putative epithelial stem cells and contributes to intestinal tumorigenesis. Cancer Res 71: 3709-3719, 2011.

18. Sellheyer K and Nelson P: Follicular stem cell marker PHLDA1 (TDAG51) is superior to cytokeratin-20 in differentiating between trichoepithelioma and basal cell carcinoma in small biopsy specimens. J Cutan Pathol 38: 542-550, 2011.

19. Johnson EO, Chang KH, Pablo Y, et al: PHLDA1 is a crucial negative regulator and effector of aurora A kinase in breast cancer. J Cell Sci 124: 2711-2722, 2011.

20. Shen YC, Hu FC, Jeng YM, et al: Nuclear overexpression of mitotic regulatory proteins in biliary tract cancer: Correlation with clinicopathologic features and patient survival. Cancer Epidemiol Biomarkers Prev 18: 417-423, 2009.

21. Hao XP, Willis JE, Pretlow TG, et al: Loss of fragile histidine triad expression in colorectal carcinomas and premalignant lesions. Cancer Res 60: 18-21, 2000.

22. Alba MM, Santibáñez-Koref MF and Hancock JM: The comparative genomics of polyglutamine repeats: Extreme differences in the codon organization of repeat-encoding regions between mammals and Drosophila. J Mol Evol 52: 249-259, 2001.

23. Coutinho-Camillo CM, Lourenço SV, Nonogaki S, Vartanian JG, Nagai MA, Kowalski LP and Soares FA: Expression of PAR-4 and PHLDA1 is prognostic for overall and disease-free survival in oral squamous cell carcinomas. Virchows Arch 463: 31-39, 2013.

24. Miraglia S, Godfrey W, Yin AH, Atkins K, Warnke R, Holden JT, Bray RA, Waller EK and Buck DW: A novel five-transmembrane hematopoietic stem cell antigen: Isolation, characterization and molecular cloning. Blood 90: 5013-5021, 1997. 
25. Yin AH, Miraglia S, Zanjani ED, et al: AC133, a novel marker for human hematopoietic stem and progenitor cells. Blood 90: 5002-5012, 1997.

26. Shmelkov SV, St Clair R, Lyden D and Rafii S: AC133/CD133/ Prominin-1. Int J Biochem Cell Biol 37: 715-719, 2005.

27. Shimada M, Sugimoto K, Iwahashi S, et al: CD133 expression is a potential prognostic indicator in intrahepatic cholangiocarcinoma. J Gastroenterol 45: 896-902, 2010.
28. Leelawat K, Thongtawee T, Narong S, et al: Strong expression of CD133 is associated with increased cholangiocarcinoma progression. World J Gastroenterol 17: 1192-1198, 2011.

29. Fan L, He F, Liu H, et al: CD133: A potential indicator for differentiation and prognosis of human cholangiocarcinoma. BMC Cancer 11: 320, 2011. 